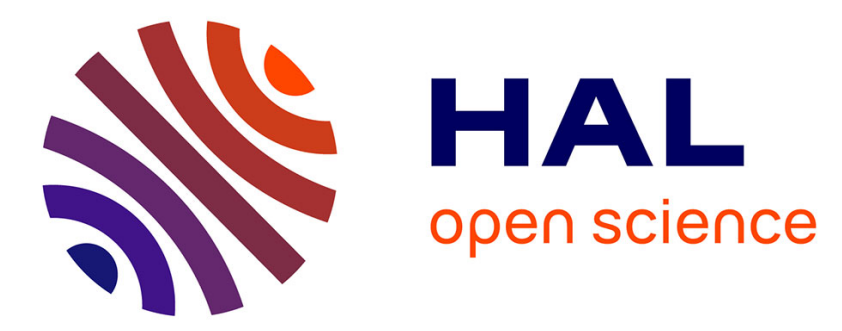

\title{
Modeling of thin structures in eddy current testing with shell elements
}

\author{
A. Ospina, L. Santandrea, Y. Le Bihan, C. Marchand
}

\section{To cite this version:}

A. Ospina, L. Santandrea, Y. Le Bihan, C. Marchand. Modeling of thin structures in eddy current testing with shell elements. European Physical Journal: Applied Physics, 2010, 52 (2), 10.1051/epjap/2010082 . hal-00634365

\section{HAL Id: hal-00634365 https://hal.science/hal-00634365}

Submitted on 21 Oct 2011

HAL is a multi-disciplinary open access archive for the deposit and dissemination of scientific research documents, whether they are published or not. The documents may come from teaching and research institutions in France or abroad, or from public or private research centers.
L'archive ouverte pluridisciplinaire HAL, est destinée au dépôt et à la diffusion de documents scientifiques de niveau recherche, publiés ou non, émanant des établissements d'enseignement et de recherche français ou étrangers, des laboratoires publics ou privés. 


\title{
Modeling of thin structures in eddy current testing with shell elements
}

\author{
Alejandro Ospina ${ }^{a}$, Laurent Santandrea, Yann Le Bihan, Claude Marchand \\ Laboratoire de Génie Electrique de Paris,CNRS UMR8507; SUPELEC; UPMC Univ Paris 06; Univ Paris-Sud 11; 11 rue \\ Joliot-Curie, Plateau de Moulon, 91192 Gif-sur-Yvette Cedex, France
}

Received: date / Revised version: date

\begin{abstract}
The modeling and design of eddy currents sensors for non-destructive testing applications, generally, requires numerical methods. Among these methods, the finite element method is one of the most used. Indeed, it presents a great capability to treat a large variety of configurations. However, in the study of eddy current testing problems, the existence of structures that have a geometrical dimension smaller than the others (thin air gaps, coatings...) will lead to difficulties related to the meshing process. The introduction of particular elements such as shell elements allows to simplify the modeling of these problems. In this paper, the shell elements are used in two different 2D axisymmetric formulations, the electric formulation $\boldsymbol{a}^{*}$ and the magnetic formulation $\boldsymbol{t}$ - $\phi$ in order to simulate the behaviour of the electromagnetic fields. The results obtained with the two formulations are compared with analytical solutions.
\end{abstract}

PACS. 81.70.-q Methods of materials testing and analysis - 07.05.Tp Computer modeling and simulation

\section{Introduction}

In the context of non-destructive testing (NDT) of metallic pieces, eddy current techniques are widely used. They have been studied by different modeling techniques, among them, the finite element method (FEM) is one of the most common. The main advantage of this method comes from its capability to deal with complex geometries. However, in eddy current NDT, thin structures frequently appear. These structures have one or two geometrical dimensions considerably smaller than the others (coatings, air gaps ...). This can present difficulties for FEM related to the meshing process, such as for example, a strong density of elements or deformed elements in the thin area and its vicinity.

A considerable effort of research was made about the modeling of electromagnetic fields by FEM in thin regions. Two main groups of solutions have been developed. All of them suppose that the behaviour of the fields is known through the thickness. The first approach uses the plane wave hypothesis and assumes that the electromagnetic fields have a tangential direction inside the thin region $[1],[2]$. In the second approach, the thickness problem is take into account during the meshing process [3],[4]. The volume of the thin region is then represented by a type of degenerated elements (shell elements) that constraints the field behaviour across the thickness by his shape functions.

Send offprint requests to:

a Present address: alejandro.ospina@lgep.supelec.fr
The shell elements avoid the use of a priori assumptions about the fields penetration inside the thin region. They allow a field description well adapted to eddy current NDT where the fields are frequently not tangential inside the thin regions. Nevertheless, the use of the shell elements implies a linear approximation of the fields along the thickness.

\section{Problem Description}

The considered geometric configuration is a classical problem in eddy current NDT (fig. 1a.): a coil placed above a conductive thin material (the target). The problem domain $\Omega$ is decomposed in three sub domains: the target material $\Omega_{c}$, which is a conductive region, the coil domain $\Omega_{0}$ and the air region. The coil carries a current density $j_{0}$.

The physical description is performed by the Maxwell reduced equations (without displacement currents):

$$
\begin{aligned}
& \operatorname{curl} \boldsymbol{h}=\boldsymbol{j}_{0}+\boldsymbol{j}, \quad \operatorname{curl} \boldsymbol{e}=-\partial_{t} \boldsymbol{b}, \\
& \operatorname{div} \boldsymbol{b}=0, \quad \boldsymbol{b}=\mu \boldsymbol{h}, \quad \boldsymbol{j}=\sigma \boldsymbol{e},
\end{aligned}
$$

where $\boldsymbol{h}$ is the magnetic field, $\boldsymbol{b}$ is the magnetic flux density, $\boldsymbol{e}$ is the electric field and $\boldsymbol{j}$ is the current density.

The thin region is characterized by a number $L$ that is the ratio between the thickness $d$ and a characteristic geometrical quantity. $L$ should be defined in two different ways: in an intuitive approach, by comparison with a $L_{g}$ dimension that is a representative geometrical dimension, 
or in an electromagnetic approach, by comparison with a $\delta$ parameter that represent the penetration of electromagnetic fields inside metallic regions. The characteristic number $L$ is defined by,

$$
L=\left\{\begin{array}{l}
d / L_{g} \text { if } L_{g}<\delta \text { or } \delta \text { not defined } \\
d / \delta \text { if } \delta<L_{g}
\end{array}\right.
$$

where $\delta=\sqrt{2 /(\omega \sigma \mu)}$ is the well-know expression of skin depth, it is defined with the physical properties of the thin region.

Two types of thin regions are considered in this paper. When the air gap between the target and the coil (the lift-off) becomes very narrow, this separation becomes the thin region, thus, it is replaced by the shell elements (fig. 1b.). In the second example, the target material has a conductive coating; this coating is modeled by the shell elements (fig. 1c.). In both, the lift-off and the coating examples, the impedance variation is studied in relation to the thin region parameter $L$.

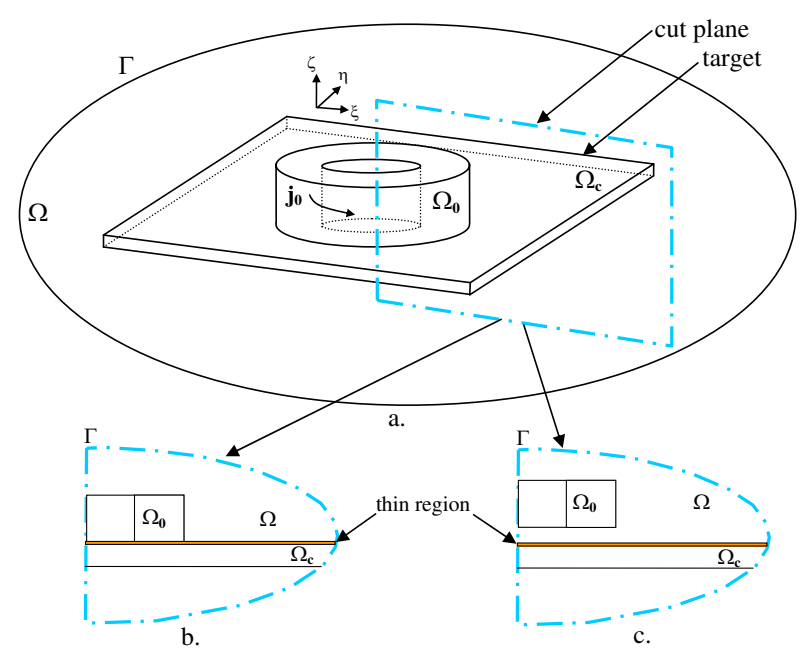

Fig. 1: a.Problem description b.Thin air gap case c.Thin coating case

\subsection{Magnetodynamic Formulations}

In order to obtain a physical model of the eddy current NDT configuration, an approach based on dual formulations of eddy currents problems has been proposed by Ren and Razek [5]. One formulation is based on the electrical field, the other on the magnetic field.

\subsubsection{Electric formulation}

The electric field $\boldsymbol{e}$ is associated with a modified magnetic vector potential $\boldsymbol{a}^{*}$, in the form $\boldsymbol{a}^{*}=-\int_{t} \boldsymbol{e} d t$. The weak formulation starts applying the Ampere's law, for $t>0$ in the harmonic regime, thus, the weak formulation is

$$
\begin{aligned}
& \int_{\Omega} \frac{1}{\mu} \operatorname{curl} \boldsymbol{a}^{\prime} \cdot \operatorname{curl} \boldsymbol{a}^{*} d \Omega+\int_{\Gamma}(\mathbf{n} \times \boldsymbol{h}) \cdot \boldsymbol{a}^{\prime} d \Gamma= \\
& \int_{\Omega} \boldsymbol{a}^{\prime} \cdot\left(\boldsymbol{j}_{0}-i \omega \sigma \boldsymbol{a}^{*}\right) d \Omega
\end{aligned}
$$

where $\boldsymbol{a}^{\prime}$ is a test function.

\subsubsection{Magnetic formulation}

The magnetic field $\boldsymbol{h}$ is decomposed in two potentials, the electric vector potential $\boldsymbol{t}$ and the magnetic scalar potential $\phi$, then $\boldsymbol{h}=\boldsymbol{t}-\operatorname{grad} \phi$. The weak form starts applying the Faraday's law to conductor materials. Thus, the weak formulation is

$$
\begin{aligned}
& \int_{\Omega_{c}} \frac{1}{\sigma} \operatorname{curl} \boldsymbol{t}^{\prime} \cdot \operatorname{curl} \boldsymbol{t} d \Omega+\int_{\Gamma_{c}}(\mathbf{n} \times \boldsymbol{e}) \cdot \boldsymbol{t}^{\prime} d \Gamma= \\
& \int_{\Omega_{c}} i \omega \mu \boldsymbol{t}^{\prime} \cdot(\operatorname{grad} \phi-\boldsymbol{t}) d \Omega,
\end{aligned}
$$

where $\boldsymbol{t}^{\prime}$ is a vector test function. Outside metallic regions, the formulation need another additional equation that verify the $\operatorname{div} \boldsymbol{b}=0$, thus the weak formulation is completed by

$$
\begin{gathered}
\int_{\Omega} i \omega \mu \operatorname{grad} \phi^{\prime} \cdot(\boldsymbol{t}-\operatorname{grad} \phi) d \Omega+ \\
\int_{\Gamma} i \omega(\mathbf{n} \cdot \boldsymbol{b}) \phi^{\prime} d \Gamma=0,
\end{gathered}
$$

where $\phi^{\prime}$ is a nodal test function.

\section{Shell elements}

The shell elements are a space degeneration of the finite elements. They are geometrical objects that have a $D-1$ geometrical dimension, where $D$ is the model geometrical dimension (in general $D=3$ or 2 ). If the problem is three-dimensional $(D=3)$, in the first stage of discretization process (meshing), the thin region is represented by a middle surface $\left(\Gamma_{s h}\right)$. Then, the edge and the nodes in $\Gamma_{s h}$ are projected in two "virtual" surfaces $\left(\Gamma_{s h}^{+}\right.$and $\left.\Gamma_{s h}^{-}\right)$. The physical behaviour inside the thin region can be represented by the shape functions in these surfaces. In the proposed examples, the device has an axisymmetrical symmetry, thus, they can be considered as a 2 dimensional model and, in consequence, the shell elements are 1 dimensional geometrical objects (lines).

Rectangular degenerated elements are used in the examples (fig. 2). These elements are similar to prismatic degenerated elements proposed in [4]. The scalar and vectorial fields are approximated in thin regions by,

$$
\begin{gathered}
\phi=\sum_{i \in N_{s h}} w_{s h_{i}}^{0} \overline{\phi_{i}}=w_{s h}^{0} \bar{\phi}, \\
\boldsymbol{t}=\sum_{k \in E_{s h}} \boldsymbol{w}_{s h_{\boldsymbol{k}}}^{1} \overline{t_{k}}=\boldsymbol{w}_{s h}^{1} \bar{t}
\end{gathered}
$$

where, $N_{s h}=\left\{N_{s h}^{+} \cup N_{s h}^{-}\right\}$and $E_{s h}=\left\{E_{s h}^{+} \cup E_{s h}^{-} \cup E_{s h}^{ \pm}\right\}$ are, respectively, the set of nodes and the set of edges (with 

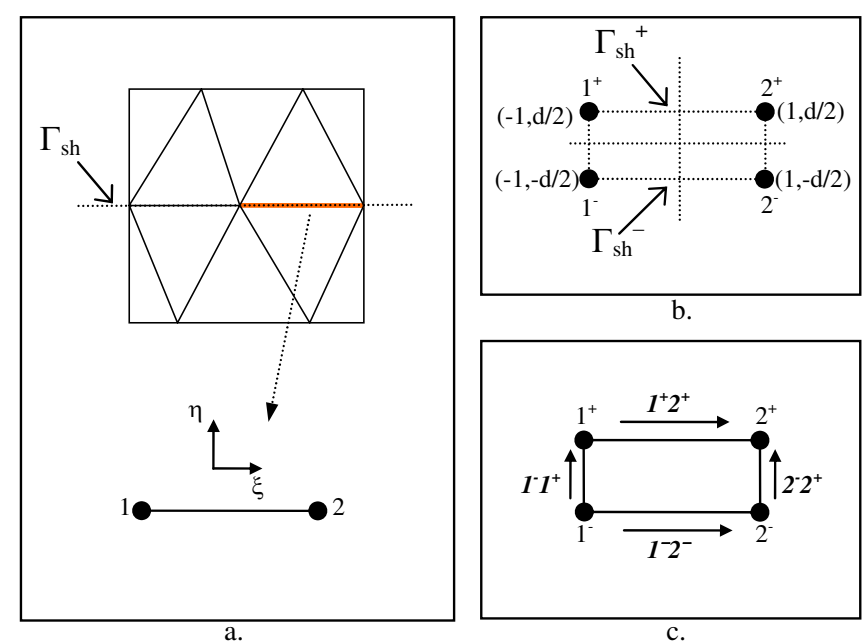

Fig. 2: 2D shell element. a. mesh b. split nodes c. split edges

$E_{s h}^{ \pm}$as the set of transversal edges) that represented the thin region.

$w_{s h}^{0}=\left\{w_{s h_{i}}^{0}, i \in N_{s h}\right\}$ is the vector of nodal shape fonctions, and,

$\boldsymbol{w}_{\boldsymbol{s h}}^{1}=\left\{\boldsymbol{w}_{\boldsymbol{s h}_{\boldsymbol{k}}}^{1}, k \in E_{s h}\right\}$ is the vector of edge shape functions. $\bar{\phi}$ and $\bar{t}$ represent, respectively, the vector of scalar potential values on the nodes and the vector of circulation values of the vector potential $\boldsymbol{t}$ along the edges that discretize the thin region.

The nodal shape fonctions are defined by:

$$
\begin{aligned}
& w_{s h_{i-}}^{0}=w_{l_{i}}^{0} \alpha^{-}, \\
& w_{s h_{i^{+}}}^{0}=w_{l_{i}}^{0} \alpha^{+},
\end{aligned}
$$

where $w_{l_{i}}^{0}$ are the shape fonctions in a line element,

$$
\begin{aligned}
& w_{l_{1}}^{0}=1 / 2(1-\xi), \\
& w_{l_{2}}^{0}=1 / 2(1+\xi) .
\end{aligned}
$$

These shape fonctions are projected on the lines $\Gamma_{s h}^{-}$and $\Gamma_{s h}^{+}$by the coefficients $\alpha^{-}$and $\alpha^{+}$defined by,

$$
\begin{aligned}
& \alpha^{-}=1 / 2-\eta / d \\
& \alpha^{+}=1 / 2+\eta / d
\end{aligned}
$$

The edge shape functions are defined by the relations,

$$
\begin{aligned}
& \boldsymbol{w}_{s h_{k}-}^{1}=\boldsymbol{w}_{l_{k}}^{1} \alpha^{-} \\
& \boldsymbol{w}_{s h_{k}+}^{1}=\boldsymbol{w}_{l_{k}}^{1} \alpha^{+}, \\
& \boldsymbol{w}_{s h_{k} \pm}^{1}=\left(\alpha^{-} \operatorname{grad} \alpha^{+}-\alpha^{+} \operatorname{grad} \alpha^{-}\right) w_{l_{i}}^{0},
\end{aligned}
$$

where the subindice $k$ identifies the edges that connect the nodes $i$ to $j$ on the surface $\Gamma_{s h}^{+}$or $\Gamma_{s h}^{-}$. The subindice $k^{ \pm}$identifies the edges that connect a node from the surface $\Gamma_{s h}^{-}$with his double over $\Gamma_{s h}^{+} . \boldsymbol{w}_{l_{k}}^{1}$ represents the edge shape function of a line element, that is defined by

$$
\boldsymbol{w}_{l_{\boldsymbol{k}}}^{1}=w_{l_{i}}^{0} \operatorname{grad} w_{l_{j}}^{0}-w_{l_{j}}^{0} \operatorname{grad} w_{l_{i}}^{0} .
$$

\subsection{Shell elements approximation in the magnetodynamic formulations}

The gradient and curl operators can be approximated in a suitable form by the nodal and edge shell functions. Thus, in the thin regions the weak formulations can be reformulated. In order to illustrate this process, the magnetodynamic $\boldsymbol{t}-\phi$ formulation are approximated. First, the gradient is changed as,

$$
\begin{aligned}
\operatorname{grad} \phi=\sum_{i \in N_{s h}} & \left(\alpha^{-} \operatorname{grad}_{s} w_{l_{i}}^{0}-\frac{w_{l_{i}}^{0}}{d} \mathbf{n}\right) \phi_{i}^{-}+ \\
& \left(\alpha^{+} \operatorname{grad}_{s} w_{l_{i}}^{0} \frac{w_{l_{i}}^{0}}{d} \mathbf{n}\right) \phi_{i}^{+},
\end{aligned}
$$

and the curl is approximated in a similar way by

$$
\begin{array}{r}
\operatorname{curl} \boldsymbol{t}=\sum_{k^{-} \in E_{s h}^{-}}\left(\alpha^{-} \operatorname{curl}_{s} \boldsymbol{w}_{\iota_{k}}^{1}-\frac{1}{d} \mathbf{n} \times \boldsymbol{w}_{\iota_{\boldsymbol{k}}}^{1}\right) \bar{t}_{k^{-}}+ \\
\sum_{k^{+} \in E_{s h}^{+}}\left(\alpha^{+} \operatorname{curl}_{s} \boldsymbol{w}_{\iota_{\boldsymbol{k}}}^{1}+\frac{1}{d} \mathbf{n} \times \boldsymbol{w}_{\iota_{\boldsymbol{k}}}^{1}\right) \bar{t}_{k^{+}}+ \\
\sum_{i^{ \pm} \in E_{s h}^{ \pm}}\left(\frac{1}{d} \operatorname{grad}_{s} w_{l_{i}}^{0} \times \mathbf{n}\right) \bar{t}_{n_{i^{ \pm}}}
\end{array}
$$

where $\mathbf{n}$ is a normal vector to $\Gamma_{s h}$. The vector

$\bar{t}_{n}=\left\{\bar{t}_{n_{i^{ \pm}}}, i^{ \pm} \in E_{s h}^{ \pm}\right\}$contains the circulation of $\boldsymbol{t}$ over the perpendicular edges to line $\Gamma_{s h}$; These edges connect the up and down nodes. Using these approximations the $\boldsymbol{t}-\phi$ formulation is transformed in a linear system in which, the unknowns are the circulation $\bar{t}_{k}$ over the edges and the scalar quantities $\bar{\phi}_{i}$ in the nodes. For example, the first term in eq. (4) related to the edges in the line $\Gamma_{s h}^{-}$is transformed,

$$
\begin{array}{r}
\int_{\Gamma_{s h}} \int_{-d / 2}^{d / 2} \frac{1}{\sigma} \sum_{\substack{j \in E_{s h}^{-} \\
k \in E_{s h} \\
i^{ \pm} \in N_{s h}}}\left[\alpha^{-} \operatorname{curl}_{s} \boldsymbol{w}_{l_{j}}^{1} \quad-\frac{1}{d} \mathbf{n} \times w_{l_{j}}^{0}\right] \\
\left(\left[\begin{array}{c}
\alpha^{-} \mathbf{c u r l}_{s} \boldsymbol{w}_{l_{k}}^{1} \\
-\frac{1}{d} \mathbf{n} \times w_{l_{k}}^{0}
\end{array}\right] \bar{t}_{k^{-}}+\left[\begin{array}{c}
\alpha^{+} \operatorname{curl}_{s} \boldsymbol{w}_{l_{\boldsymbol{k}}}^{1} \\
+\frac{1}{d} \mathbf{n} \times w_{l_{k}}^{0}
\end{array}\right] \bar{t}_{k^{+}}+\right. \\
\left.\left[\frac{1}{d} \operatorname{grad}_{s} w_{l_{i}}^{0} \times \mathbf{n}\right] \bar{t}_{n_{i^{ \pm}}}\right) d \eta d \Gamma .
\end{array}
$$

In a similar form, the first term in eq. (5) related to the nodes located on the line $\Gamma_{s h}^{-}$, is transformed,

$$
\begin{gathered}
\int_{\Gamma_{s h}} \int_{-d / 2}^{d / 2} i \omega \mu \sum_{\substack{i \in N_{s h}^{-} \\
j \in N_{s h}}}\left[\alpha^{-} \operatorname{grad}_{s} w_{l_{i}}^{0}-w_{l_{i}}^{0} / d\right] . \\
\left(\left[\begin{array}{c}
\alpha^{-} \operatorname{grad}_{s} w_{l_{j}}^{0} \\
-w_{l_{j}}^{0} / d
\end{array}\right] \phi_{j^{-}}+\left[\begin{array}{c}
\alpha^{+} \operatorname{grad}_{s} w_{l_{j}}^{0} \\
w_{l_{j}}^{0} / d
\end{array}\right] \phi_{j^{+}}\right) d \eta d \Gamma .(18)
\end{gathered}
$$

Remarks that the use of shell elements transforms the integral domains: in a 2D case, the surface integrals are reduced to line integrals, and in a 3D problem, the volume integrals are changed by surface integrals. 


\section{Results}

In the proposed examples, the geometrical dimensions are taken from the TEAM Workshop problem n. 15-1 [6], these dimensions are synthetized in table (1). The characteristic geometrical parameter $L_{g}$ is the inner radius of the coil and $\delta \approx 3.0 \mathrm{~mm}$ (calculated with the problem parameters). The test problem is solved using the two bidimensional formulations previously exposed. In the first stage of the modeling process, a triangular mesh is constructed. This mesh is highly refined to reduce the error originated by triangular elements. After that, the shell elements are inserted in the thin region. Thus, the error is strongly dependent on the shell elements.

Table 1: Problem Parameters

\begin{tabular}{ll}
\hline coil parameters & \\
\hline inner radius & $6.15 \mathrm{~mm}$ \\
outter radius & $12.3 \mathrm{~mm}$ \\
heigth & $6.15 \mathrm{~mm}$ \\
number of turns & 3790 \\
\hline target parameters & lift-off example \\
\hline thickness & $12.22 \mathrm{~mm}$ \\
conductivity & $30.6 \times 10^{6} \mathrm{~S} / \mathrm{m}$ \\
relative permeabiltity & 1 \\
\hline target parameters & coating example \\
\hline thickness & $12.22+d \mathrm{~mm}$ \\
coating conductivity & $30.6 \times 10^{6} \mathrm{~S} / \mathrm{m}$ \\
substrate conductivity & $1 \times 10^{6} \mathrm{~S} / \mathrm{m}$ \\
relative permeabiltity & 1 \\
\hline other parameters & \\
\hline frequency & $900 \mathrm{~Hz}$ \\
\hline
\end{tabular}

In the first example, the lift-off case, $d$ varies in the interval $[1 \mu \mathrm{m}, 1 \mathrm{~mm}]$. The characteristic parameter $L$ is defined by eq. (2), here $\delta$ is not defined $(\sigma=0)$. In the second example, the coating case, $d$ varies in the same interval than in the lift-off case, and the characteristic parameter $L$ is defined by eq. (2), here $\delta<L_{g}$. The analytical results are calculated by Dodd and Deeds model [7]. The relative errors are calculated by,

$$
\begin{aligned}
& R_{\text {error }}=\frac{\left|R_{\text {model }}-R_{\text {analytical }}\right|}{\left|Z_{\text {analytical }}\right|} \\
& X_{\text {error }}=\frac{\left|X_{\text {model }}-X_{\text {analytical }}\right|}{\left|Z_{\text {analytical }}\right|}
\end{aligned}
$$

where $R_{\text {analytical }}, X_{\text {analytical }}$ and $Z_{\text {analytical }}$ are the analytical resistance, reactance and impedance, respectively. $R_{\text {model }}$ and $X_{\text {model }}$ are the resistance and reactance obtained by the dual formulations. The resistance, the reactance and consequently the impedance are associated with the coil. These relative errors are illustrated, for lift-
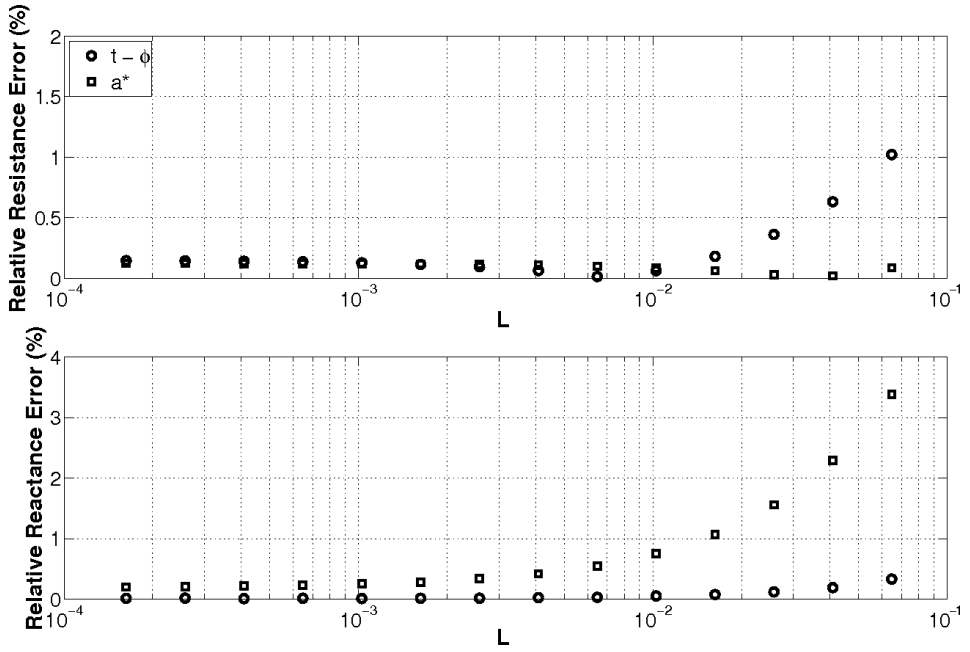

Fig. 3: Relative Errors. Lift-off case.
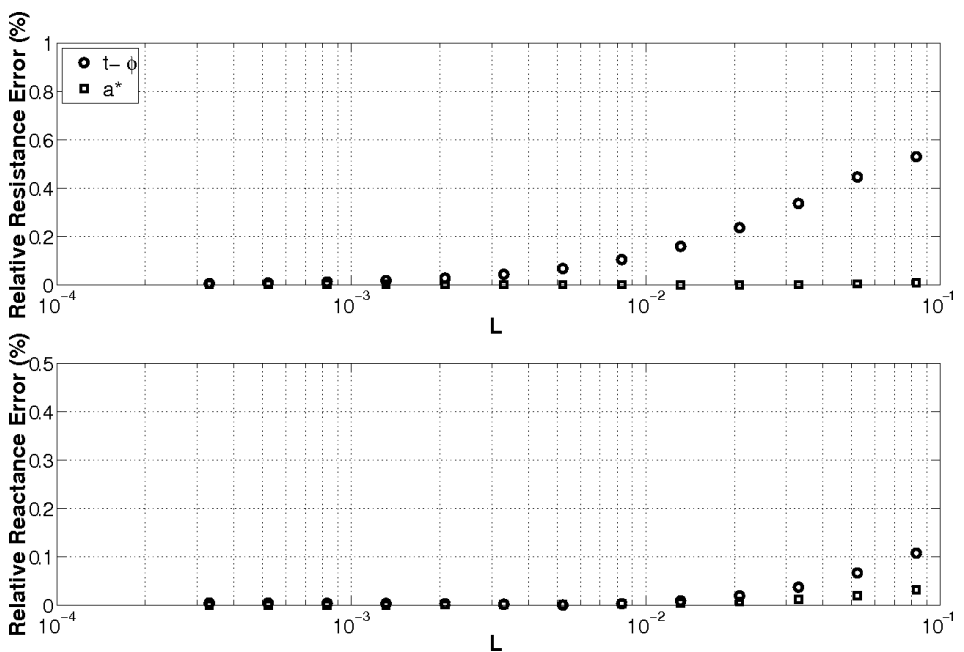

Fig. 4: Relative Errors. Coating case.

off case in fig (3) and for coating case in fig (4).

The $\boldsymbol{a}^{*}$ formulation verifies in a strong way the Faraday's law and in a weak form the Ampere's law, by contrast, the $\boldsymbol{t}-\phi$ formulation verifies in a strong way the Ampere's law and in a weak form the Faraday's law. Thus, the results expected for the resistance will be better calculated in the $\boldsymbol{a}^{*}$ formulation in comparison with the $\boldsymbol{t}-\boldsymbol{\phi}$ formulation. By symmetry, the magnetic field and the associated reactance will be better calculated in the $\boldsymbol{t}-\phi$ formulation than in $\boldsymbol{a}^{*}$.

The results show than, in the lift-off case, the relative error on the reactance, calculated by $\boldsymbol{a}^{*}$, is more important than in the $\boldsymbol{t}-\phi$ formulation. In an analog situation, in the coating case, the relative error on the reactance, calculated by $\boldsymbol{t}-\phi$ formulation, is more important than in the $\boldsymbol{a}^{*}$ formulation. The relative resistance error obtained by the $\boldsymbol{a}^{*}$ formulation shows a better approximation for both lift-off and coating cases, this is different from expected results. The explanation comes from the fields approxi- 
mation which is made by the linear shape functions in the shell elements and the influence of these elements on the calculation of reactance and resistance values. In the lift-off case, the shell elements have a direct influence in the reactance value and indirectly in the resistance value, in the coating case, they have a direct influence in both resistance and reactance calculations.

In the lift-off case, the use of $\boldsymbol{a}^{*}$ formulation implies a linear variation of the electric field $e$ and, in metallic regions (target material with a triangular mesh), a linear variation of the current density. The magnetic field $\boldsymbol{h}$, calculated by the curl operator, is constant by element and the relative error is quite important on the reactance. By contrast, the resistance is more accurately calculated (triangular elements). In $\boldsymbol{t}-\phi$ formulation, the behaviour of shell elements is symmetrical: the magnetic field $\boldsymbol{h}$ has a linear variation along the lift-off thickness. The variation is more realistic, and the relative error on the reactance is less important. The current density is constant by element inside metallic regions (triangular elements) since it is calculated as the curl of the electric vector potential $(\operatorname{curl} t)$. Thus, the resistance has a more important relative error in comparison with $\boldsymbol{a}^{*}$ formulation.

In the coating case, the electric field $\boldsymbol{e}$ calculated by the $\boldsymbol{a}^{*}$ formulation has a linear variation along the coating thickness (shell elements). The current density $\boldsymbol{j}$ will be linear in all metallic domains (triangular and shell elements). This is a good approach to physical reality, especially if the skin depth is very large compared with coating thickness. On the other hand, in $t-\phi$ formulation the current density $j$ is constant inside the shell elements since it is calculated by the curl operator $(\operatorname{curl} t=j)$. This approximation is less adapted to the physical reality. Consequently, the relative error on the resistance is greater than the one obtained with the $\boldsymbol{a}^{*}$ formulation. In this case, the calculation of reactance is very similar in both formulations: outside the metallic regions (coating and substrate) the magnetic field is constant by element, in this way, the relative reactance error has a comparable value (differences ¡0.1\%) and behaviour for both electrical and magnetic formulations.

\section{Conclusions}

In both cases, the lift-off and the coating cases, the results obtained with the shell element dual formulations exbihit a good agreement compared with the analytical results (Relative errors $<4 \%$ ). The application of the shell elements in magnetodynamic formulations represents an interesting solution when the meshing with classical elements is impossible to realize or requires a high number of elements.

\section{References}

1. P. Dular, C. Geuzaine, "Modeling of Thin insulating Layers With Dual 3-D Magnetodynamic Formulations", IEEE
Transactions on Magnetics, vol. 39,no. 3,(May 2003), pp. 1139-1142.

2. C., Geuzaine, P. Dular, W. Legros, "Dual formulations for the modeling of thin electromagnetic shells using edge elements," IEEE Transactions on Magnetic vol. 36, no. 4, (Jul 2000) pp. 799-803.

3. Y. Choua, L. Santandrea, Y. Le Bihan, C.Marchand,"Thin CrackModeling in ECT with Combined Potential Formulations," IEEE Transactions on Magnetics,vol. 43, no. 4,(Apr 2007) pp. 1789-1792.

4. Z. Ren,"Degenerated Whitney Prism Elements - General Nodal and Edge shell Elements for Field Computation in Thin Structures,"IEEE Transactions on Magnetics,vol. 34,no. 5, (Sept 1998), pp. 2547-2550.

5. Z. Ren, A. Razek, "Computation of 3D electromagnetic field using differential forms based elements and dual formulations", Int. J. Numer. Model. , vol. 9,no. 3,(1996), pp. $81-98$.

6. S.K. Burke, "A Benchmark problem for computation of $\Delta \mathrm{Z}$ in eddy current non-destructive evaluation (NDE)", J. Nondestruct. Eval., vol. 7,no. 1/2, (1988), pp. 35-41.

7. C. V. Dodd, W. E. Deeds, "Analytical Solutions to EddyCurrent Probe-Coil Problems", J. Appl. Phys., vol. 39,no. 6, (1968), pp. 2829-2838. 